\title{
Editorial Statement: Social Issues and Policy Review
}

\author{
Jolanda Jetten \\ University of Queensland
}

\section{Naomi Ellemers}

University of Utrecht

As the incoming coeditors of Social Issues and Policy Review, we appreciate this opportunity to share our view on where we hope to take SIPR in the next couple of years. Before doing so, we would like to thank the previous editors, Lou Penner and Rudy Mendoza-Denton, for their excellent work. They handed over a journal that is in outstanding shape and we are extremely grateful for this.

More specifically, under the stewardship of Lou and Rudy (and Rupert Brown and Sam Gaertner before them), SIPR has seen a spectacular rise in its impact over the last years and it now ranks amongst the top journals in psychology, with an impact factor of 6.14. It has gained an international reputation as an outlet for the review of research programs of the highest standard. The journal also occupies a distinctive niche in the discipline in that it is one of the few outlets where researchers connect work in a wide variety of fields to policy. For this reason, contributions to the journal tend to engage an unusually broad and diverse constituency of readers. We see this diversity as one of the journal's great strengths. It is also a quality that we as an editorial team will seek to nurture and preserve.

We also realize, however, that we are facing new challenges when it comes to ensuring that policy has the best possible evidence base. Arguably, at a time of "fact-free politics" and the accumulation of "fake truths," it has become more important but also less straightforward for social scientists to explain their work to broad audiences and to specify the policy implications of their findings. Another challenge lies in the fact that, for readers new to an area, it is not always easy to judge which insights are robust and which ones are merely isolated findings (that may or may not connect to the broader body of work). As a result, given the vast amount of research that is being conducted and published in different outlets, the big picture may get lost and it becomes more difficult to see the forest for the trees-especially for those working in a different area. 
We believe that SIPR is ideally placed to deal with these challenges. Providing overviews of large-scale research programs, combining conclusions from experimental and applied studies, and spelling out the theoretical and practical implications of accumulated bodies of findings should offer a constructive way forward in current debates about the value of science and academic research in the social sciences and policy fields more generally.

In terms of the work we aim to attract during our 3-year term, within the broader context of the established and formal mission of the journal, we particularly hope to publish work that speaks to the key social issues of our time. In particular, we emphasize the added value of SIPR as an outlet that:

(1) provides specialist as well as nonspecialist readers with comprehensive and balanced reviews of research that addresses current social issues with clear policy implications. This implies that-instead of offering academic literature reviews - contributions address a broad audience of nonspecialist readers, including scholars working in other disciplines and policy makers seeking information about what our field has to offer,

(2) links and connects research findings to policy whereby policy recommendations flow logically from a strong and solid research evidence base. This requires that authors consider the policy implications of the theory and evidence they review in an integrated way throughout their contributioninstead of only addressing this in a single section or specific paragraph,

(3) communicates accumulated knowledge bases whereby research insights are truthfully communicated by the experts themselves in a way that is accessible to lay audiences. To achieve this aim, we invite authors who are established researchers in their area of expertise to provide an accessible overview of a mature program of research, highlighting their own (published) work, but also integrating this with other relevant developments.

We are strongly committed to the mission and scope of this journal, and are looking forward to the work ahead. We hope that you will continue to support $S I P R$ over the next couple of years, and look forward to serving you as readers or contributors of this outlet. 